\title{
AQUISIÇÃO DE REPERTÓRIO DISCRIMINATIVO AUDITIVO EM ESQUEMA MÚLTIPLO: EXPLORANDO PROCEDIMENTO PARA ESTUDO DA FORMAÇÃO DE CLASSES FUNCIONAIS EM RATOS
}

\section{ACQUISITION OF CONCURRENT DISCRIMINATION WITH MULTIPLE SCHEDULES: EXPLORING A PROCEDURE FOR FUNCTION CLASS FORMATION IN RATS}

\author{
Thiago Dias Costa, Romariz da Silva Barros e Olavo de Faria Galvão \\ UNIVERSIDADE FEDERAL DO PARÁ, BRASIL \\ Maria de Jesus Dutra dos Reis \\ UNIVERSIDADE FEDERAL DE SÃO CARLOS, BRASIL
}

\begin{abstract}
RESUMO
Estudos sobre a formação de classes de estímulos usualmente requerem o treino de discriminações de linha de base que incluem múltiplas discriminações simples e/ou condicionais inter-relacionadas. O objetivo do presente estudo foi replicar a fase de aquisição de discriminações de linha de base de um estudo anterior com ratos como sujeitos, com mudanças de procedimento objetivando tornar mais eficiente o treino de linha de base. Seis ratos albinos (Rattus norvegicus) foram submetidos a treino de discriminaçōes simples sucessivas, com estímulos auditivos, em sessões de longa duração. Dois estímulos auditivos (A1 e B1) foram correlacionados a esquema de razão variável (VR) e dois outros estímulos (A2 e B2) foram correlacionados a esquema de reforço diferencial de taxas baixas (DRL). Cinco dos seis ratos demonstraram aquisição das discriminações, desenvolvendo dois padrões distintos de responder (taxas altas no esquema VR e taxas baixas no esquema DRL) sob controle dos estímulos auditivos. Este trabalho sugere que o emprego de esquemas múltiplos com estímulos auditivos em sessões de longa duração pode representar uma alternativa rápida e eficaz na formação de uma linha de base necessária aos estudos de formação de classes funcionais com estímulos auditivos e com ratos como sujeitos.
\end{abstract}

Palavras-chave: razão variável, reforço diferencial de taxas baixas, estímulos auditivos, Rattus novergicus

\begin{abstract}
Studies on stimulus class formation usually require training of baseline discriminations that comprise multiple interrelated simple and/or conditional discriminations. The purpose of the present study was to replicate the acquisition of baseline discriminations of a previous study with rats as subjects, introducing changes in the procedure in order to make more efficient the baseline training. Six albino rats (Rattus norvegicus) were exposed to a successive simple discrimination training procedure with auditory stimuli in long duration sessions. Two different auditory stimuli (A1 and B1) were correlated to a variable ratio schedule (VR) and two other stimuli (A2 and B2) were correlated to differential reinforcement of low rates schedule (DRL). Five out of the six rats acquired the discriminations showing two distinct patterns of response (high rates in the VR schedule and low rates in the DRL Schedule) under control of the auditory stimuli. Results suggest that the use of a multiple schedule with auditory stimuli in long duration sessions may be a fast and efficient alternative to build a baseline repertoire in studies on functional class formation with auditory stimuli and rats as subjects.
\end{abstract}

Key-words: variable ratio, differential reinforcement of low rates, auditory stimulus, Rattus norvegicus

A formação de classes de estímulos é procedimento. É possível que a verificação de difícil de ser demonstrada em sujeitos não classes de equivalência envolva pré-requisitos humanos, em parte, por problemas de comportamentais que humanos, mas não

\footnotetext{
$1 \mathrm{O}$ presente trabalho é parte da dissertação de mestrado do primeiro autor, sob orientação do terceiro autor e co-orientação do quarto. Este trabalho foi parcialmente financiado pela Coordenação de Aperfeiçoamento de Pessoal de Nível Superior, através da bolsa de mestrado ao primeiro autor. Endereço para correspondência: Thiago Dias Costa, Departamento de Psicologia Experimental, Universidade Federal do Pará, Campus Universitário do Guamá, Rua Augusto Corrêa, 01, CEP 66075-110 - Caixa Postal 479-Belém - PA. E-mail: thiago_dc@yahoo.com.
} 
animais de laboratório, possuem em sua história pré-experimental (Dube, Mcllvane, Callahan, \& Stoddard, 1993). Desta forma, há a necessidade de identificar e descrever aspectos metodológicos que possam ser, direta ou indiretamente, responsáveis pelas aparentes diferenças encontradas entre espécies, antes que se possa afirmar categoricamente que existem diferenças intransponíveis entre o desempenho humano e o de outros animais no que diz respeito à formação de classes de equivalência.

$\mathrm{Na}$ grande maioria das vezes, a formação de classes tem sido demonstrada em estudos utilizando procedimento de discriminação condicional. Um aspecto crítico nestes estudos tem sido a emergência de desempenhos sem uma história imediata de reforçamento. Seguindo-se a aprendizagem de discriminaçôes condicionais, observam-se relações entre estímulos que emergem sem treino explícito: (1) relações condicionais dos estímulos com eles mesmos (reflexividade); (2) relações condicionais bidirecionais entre dois diferentes estímulos (simetria) e (3) relações condicionais entre diferentes estímulos através de relações com estímulos modelo ou comparações comuns (transitividade). Essas relações emergentes documentam propriedades das relaçôes treinadas que as caracterizam como relações de equivalência (de Rose, Mcllvane, Dube, Galpin, \& Stoddard, 1988; Sidman, 1971; Sidman, \& Tailby, 1982).

A despeito do grande número de estudos com participantes humanos, a descrição de relações emergentes de equivalência utilizando o procedimento de treino por discriminação condicional em sujeitos não humanos ocorreu, até o presente momento, em somente duas diferentes espécies (Kastak, Schusterman \&
Kastak, 2001; Schusterman \& Kastak, 1993, com leões marinhos; Manabe, Kawashima, \& Staddon, 1995, com periquitos).

$\mathrm{Na}$ busca por procedimentos alternativos para demonstração de classes em sujeitos não humanos, Vaughan (1988) discutiu a possibilidade da formação de classes de estímulos, posteriormente denominada Classes Funcionais de Estímulos, utilizando o procedimento de reversóes repetidas de discriminações simples. Em seu trabalho, uma seqüência de 40 slides de árvores era apresentada a pombos. Os 40 estímulos foram arbitrariamente divididos em grupos de 20, com um dos grupos funcionando como $S_{+}$ (positivamente correlacionados com reforçamento) e o outro grupo funcionando como S- (correlacionado com extinção da resposta de bicar) e eram apresentados em ordem imprevisível para os sujeitos. Os pombos eram, portanto, condicionados a bicar os estímulos do Grupo $S_{+}$e a não bicar os estímulos do Grupo S-. Após a aquisição das discriminações, as contingências de reforçamento eram revertidas e os membros do grupo anteriormente correlacionados com a extinção passavam a exercer a função de $S+$ e vice-versa. Depois de repetidas reversões das contingências entre os grupos, os pombos começaram a mudar suas respostas para todos os membros de um dos grupos depois de terem experimentado a reversão da contingência com alguns poucos estímulos de cada grupo. Assim, a cada reversão das contingências, os slides apresentados no começo de uma sessão prediziam a reversão das contingências para os slides apresentados no restante da sessão.

Através desse trabalho, Vaughan (1988) mostrou que pombos podem classificar um grande grupo de estímulos em dois subgrupos de 
estímulos funcionalmente equivalentes, baseados somente no compartilhamento das histórias de reforçamento relacionadas a estes estímulos. Para o autor, esta seria uma demonstração da efetividade deste procedimento em produzir classes de estímulos em sujeitos não-humanos.

Sidman (1994, 2000), discutindo o trabalho de Vaughan, reconheceu que, matematicamente, a formação de classes de estímulos, seja baseada em discriminaçôes simples ou condicionais, envolve relações de equivalência, restando saber se comportamentalmente o mesmo se aplica.

A questão foi respondida por Kastak et al (2001) que treinaram relaçōes arbitrárias entre estímulos visuais via procedimento de repetidas reversões de discriminações simples com leões marinhos e, posteriormente, testaram as possíveis emergências de desempenho proveniente deste treino empregando o procedimento de discriminação condicional (tipicamente usado em experimentos padrão de formação de classes de equivalência). Os dados mostraram que as relações estabelecidas em um tipo de procedimento podem ser observadas no outro tipo.

Pesquisas sobre a formação de classes no contexto de reversōes de discriminação simples têm, portanto, uma contribuição potencial para essa área de estudos, no sentido de esclarecer, por exemplo, parte da controvérsia sobre a capacidade de formação de classes em organismos não humanos. Estudos como os acima descritos precisam ser replicados nas mesmas e em outras espécies, mas há uma série de entraves para o emprego do procedimento de reversóes repetidas de discriminaçōes simples com animais de laboratório de vida relativamente curta como ratos. $\mathrm{O}$ primeiro deles se refere ao tempo despendido para a conclusão do procedimento. Estima-se que o tempo necessário para realizar o treino dos animais por Vaughan (1988) tenha ultrapassado 3 anos, o que inviabilizaria uma replicação direta com ratos.

O segundo entrave é o emprego de estímulos visuais para este tipo de sujeito. Iversen $(1993,1997)$ relatou a necessidade de um treino extensivo para que ratos desenvolvessem um desempenho preciso em discriminaçōes com estímulos visuais.

O terceiro entrave pode estar intimamente ligado ao primeiro. Além do número de sessōes necessárias para que os sujeitos alcançassem o desempenho desejado, o emprego de um grupo de estímulos positivos e outro de negativos pode ter aumentado ainda mais a duração de cada sessão experimental. Isso porque, a apresentação de um estímulo designado como negativo iniciava uma contingência em que respostas ao estímulo postergavam a apresentação da próxima tentativa, alongando a sessão.

Assim, uma primeira meta a ser alcançada com animais de laboratório de vida curta como ratos é o estabelecimento de um responder discriminado com um procedimento de reversões repetidas de discriminações simples que seja relativamente rápido.

$\mathrm{Na}$ literatura recente, há procedimentos que poderiam ser adotados para contornar as dificuldades inerentes às reversōes repetidas de discriminaçôes simples com ratos (D'Amato \& Salmon, 1984; Dube et al., 1993; Harrison, 1988; Ferrara, Todorov, Azzi, \& Oliveira-Castro, 1983; Reis e Costa, 2002).

Com relação aos estímulos empregados para este tipo de procedimento, Dube, Callahan e McIlvane (1993), Harrison (1988) e D'Amato e Salmon (1984) demonstraram que ratos são capazes de responder de forma discriminada a estímulos auditivos quando estes 
são emitidos próximos às chaves de resposta. No trabalho de Dube, Callahan et al. (1993), seis estímulos auditivos, apresentados sucessivamente, foram arbitrariamente divididos em dois grupos. Os ratos eram treinados a pressionar a barra de resposta na presença dos estímulos pertencentes ao grupo designado como positivo e a não responder na presença dos estímulos do grupo negativo. Uma vez que o desempenho se estabelecia, as condições eram revertidas e o grupo de estímulos designado como positivo passava a ser correlacionado com extinção e vice-versa. Os autores relataram que o responder discriminado a estímulos auditivos pode ser estabelecido com ratos nesta condição.

Como alternativa ao uso de estímulos positivos e negativos, Reis (1997) descreveu a utilização de um procedimento de discriminação simples onde os sujeitos respondiam sob dois diferentes esquemas de reforçamento: Razão Variável (VR) e Reforçamento Diferencial de Taxas Baixas (DRL). Estes esquemas têm sido descritos tradicionalmente na literatura como seletores de taxas distintas de respostas, respectivamente taxas altas e taxas baixas (ver, por exemplo, Ferster e Skinner, 1957). Desta forma, a condição experimental na qual os sujeitos devem responder a um grupo de estímulos e não responder a outro grupo de estímulos seria substituída por uma condição onde um grupo de estímulos é correlacionado à vigência do esquema de reforçamento VR enquanto o outro grupo é correlacionado ao esquema DRL.

A despeito do procedimento descrito por Reis (1997) ter se mostrado eficiente para o estabelecimento de respostas discriminativas durante a linha de base, a autora relata que os sujeitos de seu trabalho já possuíam história experimental prévia com os mesmos estímulos.

Além disso, o procedimento descrito por ela apresentava algumas características que podem ter tornado extenso o treino para estabelecimento das discriminações de linha de base e posteriores testes de formação de classes de estímulos: (1) a quantidade de treino do responder sob controle discriminativo de cada estímulo era desigual, uma vez que as respostas sob controle discriminativo dos estímulos iniciais continuavam a ser reforçadas quando novas relaçôes de controle eram estabelecidas de maneira que, ao final do treino, as relaçóes inicialmente estabelecidas tinham sido muito mais densamente reforçadas do que as mais recentes; (2) todo o treino do responder em VR antecedia o treino do responder em DRL, o que pode ter determinado dificuldades para estabelecer o responder em DRL; (3) por fim, a curta duração das sessões pode ter determinado um grande número de sessões necessárias para o estabelecimento da linha de base, o que poderia comprometer testes subseqüentes de formação de classes considerando o tempo de vida útil de um rato de laboratório.

Desta forma, o objetivo do presente trabalho foi investigar a possibilidade de treinar ratos a responder de forma discriminada a estímulos auditivos, empregando um procedimento de discriminações simples sucessivas em sessões de longa duração com o objetivo de maximizar o tempo despendido na coleta. Sessões de longa duração têm sido descritas como eficientes para diminuir o tempo de treino com ratos e pombos em tarefas de discriminação simples (Reis e Costa, 2002; Ferrara et al., 1983).

Em atenção aos aspectos mencionados anteriormente, foram empregados neste 
trabalho sujeitos experimentalmente ingênuos. Além disso, modificações de procedimento foram introduzidas de maneira a garantir o equilíbrio da quantidade de treino programado para o responder sob controle discriminativo de cada estímulo, além da alternância de treino de responder em VR e DRL ao longo da fase de aquisição dos conjuntos de discriminações.

Assim, o mesmo aparato utilizado por Dube, Callahan et al. (1993) foi utilizado, mas programando um procedimento através do qual o responder pudesse ser reforçado na presença de todos os diferentes estímulos. Esta mudança de procedimento permitiu investigar o desempenho dos sujeitos em condições em que nenhum dos componentes fosse correlacionado com extinção. Para tanto, foi delineado um esquema múltiplo no qual dois conjuntos de dois estímulos foram correlacionados a dois esquemas de reforçamento geradores de padróes distintos de respostas como descrito por Reis (1997).

Um procedimento efetivo que possibilite um treino discriminativo rápido, com quatro ou mais estímulos auditivos e dois padrões de resposta numa mesma sessão, pode representar um primeiro passo para o estudo de formação de classes funcionais com repetidas reversões de discriminações simples com animais de laboratório de vida curta como ratos.

Além da relevância no que diz respeito à busca de contingências de treino econômico e com pouca ou nenhuma digressão de controle no estabelecimento de linha de base para estudos sobre a formação de classes com animais, o presente estudo é relevante no sentido de dar continuidade à exploração do uso de estímulos auditivos em treino discriminativo com animais. Pouco tem sido efetivamente publicado a esse respeito tanto na literatura brasileira quando na literatura especializada internacional.
MÉTOdo

\section{Sujeitos}

Foram utilizados seis ratos albinos Wistar machos, experimentalmente ingênuos, reduzidos a $80 \%-85 \%$ de seu peso em condiçôes de livre acesso a alimento e água. Os animais tinham livre acesso a água na gaiola viveiro, fora da condição experimental.

\section{Equipamento e Estimulos}

As sessões foram conduzidas com os sujeitos em suas respectivas gaiolas viveiros, construídas de acrílico transparente, com $46 \mathrm{~cm}$ de comprimento por $26 \mathrm{~cm}$ de largura, e $21 \mathrm{~cm}$ de altura. Durante as sessôes experimentais, a grade removível que fechava a abertura superior da gaiola viveiro era retirada e, em seu lugar, era colocada uma chapa de acrílico de 46 X $26 \mathrm{~cm}$ sob a qual estavam fixadas duas colunas de metal, com $6 \mathrm{~cm}$ de comprimento, por $5 \mathrm{~cm}$ de largura e $15 \mathrm{~cm}$ de altura, separadas entre si por $36 \mathrm{~cm}$ e eqüidistantes das bordas. As colunas se projetavam para o interior da gaiola, afixadas à chapa de acrílico. Numa das colunas localizada no lado esquerdo da caixa, ficava uma barra de respostas. Imediatamente acima desta chave estava colocado um auto-falante. Um comedouro, onde pelotas de alimentos de 45 mg eram depositadas, se encontrava na parte inferior desta mesma coluna. Desta forma, autofalante, chave de resposta (Barra) e comedouro ficavam localizados na mesma coluna. Todos os eventos programados foram apresentados e registrados por um computador MacIntosh Plus. Este equipamento para treino de discriminação auditiva foi desenvolvido no centro de pesquisa E. K. Shriver Center for Mental Retardation da Universidade de Massachusetts (Waltham, MA, USA). 
Os estímulos auditivos eram quatro sons assim nomeados: White Noise (WN), Clicker (CLK), um tom de 2000-Hz "square-wave" $(2 \mathrm{~K})$ e uma reprodução da voz de um ser humano do sexo feminino dizendo "One" (ONE). Estes estímulos foram apresentados via circuito de auto-falantes externos do MacIntosh como repetidos segmentos de 0.4 s de duração, ajustados em $75 \pm 1$ dB SPL. A designação dos diferentes estímulos auditivos como A1, A2, B1 e B2 variou para os diferentes sujeitos experimentais. Um quinto estímulo, "DAWN" foi usado durante o treino ao comedouro.

\section{Procedimento}

As sessões experimentais eram conduzidas diariamente e consistiam em treino de discriminaçôes simples sucessivas. Pelotas de alimentos de $45 \mathrm{mg}$ eram apresentadas como conseqüência de acordo com o esquema vigente na presença de cada estímulo. Cada sessão foi programada para ser concluída após a execução de 360 tentativas ou 3 horas de sessão, o que ocorresse primeiro. Todos os sujeitos foram submetidos ao treino preliminar que consistiu no treino ao comedouro, no procedimento automatizado de modelagem da resposta de pressão à barra e no treino para adaptação do responder aos esquemas utilizados.

Após o treino de pressão à barra, os sujeitos foram submetidos a um procedimento de treino (ver Reis, 1997) que paulatinamente levavam o responder a alcançar os valores finais dos esquemas de reforçamento correlacionados com cada um dos estímulos auditivos (A1 e B1 correlacionados com o esquema de reforçamento VR 15; A2 e B2 correlacionados com DRL 5s).

Treino ao comedouro. $\mathrm{O}$ treino era iniciado com uma sessão de adaptação do sujeito ao dispensador de alimentos e à condição experimental. A barra não estava presente no aparato. Cinco pelotas de alimento eram colocadas no comedouro, estando disponíveis para o sujeito no início da sessão. Pelotas de alimento eram dispensadas, não contingente às respostas do sujeito, em tempo variável de 40 a $70 \mathrm{~s}$, até que vinte pelotas fossem dispensadas.

Após este período, 52 outras pelotas foram dispensadas, sendo que cada acionamento de comedouro foi imediatamente precedido por um estímulo sonoro, (DAWN), apresentado durante $3 \mathrm{~s}$.

Modelagem da resposta de pressão à barra. $\mathrm{Na}$ segunda sessão, a barra foi colocada no aparato experimental e cinco pelotas de alimento foram previamente dispostas sobre o comedouro. Os sujeitos eventualmente pressionaram a barra no curso de seu comportamento exploratório; cada pressão à barra foi seguida pela apresentação de comida, em CRF, até que 80 pelotas de comida fossem apresentadas. Não havia nenhum som programado para essa sessão.

Modelagem do responder em VR15. O treino prosseguiu com a modelagem do responder sob esquema de Razão Variável 15 (VR 15), cujo estímulo associado era A1. Nesta condição experimental, foram utilizados progressivamente os seguintes valores do esquema de reforçamento: VR 2, VR 5, VR 8, VR 10, VR 12 e VR 15.

Cada tentativa se iniciava com a apresentação do estímulo auditivo A1 por até $30 \mathrm{~s}$; caso o sujeito completasse a exigência do esquema de VR em vigor, o estímulo era descontinuado, o reforço apresentado e seguido por um Intervalo entre Tentativas (IET) de $5 \mathrm{~s}$; caso o requerimento não fosse cumprido nos 30s de apresentação do estímulo, o mesmo era interrompido sem apresentação de qualquer 
reforço e um período de IET 5 s tinha início. Respostas emitidas pelos sujeitos nos últimos 2 $s$ do Intervalo entre Tentativas postergavam a apresentação da próxima tentativa por mais $5 \mathrm{~s}$.

Nesta fase de modelagem, cada sessão era dividida em blocos de 60 tentativas, totalizando seis blocos em uma sessão. As primeiras seis tentativas da primeira sessão foram programadas em CRF, as 54 tentativas seguintes foram programadas em VR 2. O critério exigido para encerrar esta condição era de que pelo menos 90\% dos reforços programados fossem obtidos. As tentativas eram repetidas caso o sujeito obtivesse, em um bloco de 60 tentativas, menos que 54 reforços. Caso este critério fosse alcançado em um bloco de tentativas, o valor seguinte do esquema (VR 5) era automaticamente apresentado no próximo bloco e assim por diante, até que 360 tentativas fossem concluídas.

A sessão experimental seguinte prosseguia com a programação de blocos de tentativas partindo do valor de VR praticado na sessão anterior.

A modelagem do responder na presença do som que sinalizava VR 15 era considerada concluída quando o sujeito obtivesse $90 \%$ ou mais dos reforços programados para uma sessão.

Modelagem do responder em DRL 5 s. Após a modelagem do responder em VR 15, a modelagem do responder em DRL 5 s tinha início. Nesta condição experimental foram usados progressivamente os seguintes valores para este esquema de reforçamento: DRL $1 \mathrm{~s}$, DRL 3 s e DRL 5 s. Cada tentativa se iniciava com a apresentação do estímulo auditivo A2 por até $30 \mathrm{~s}$, correlacionado com o esquema DRL; caso o sujeito completasse a exigência do esquema de DRL em vigor, o estímulo era descontinuado, o reforço apresentado e seguido por um IET de $5 \mathrm{~s}$; caso o requerimento não fosse cumprido nos 30 s de apresentação do estímulo, o mesmo era interrompido sem apresentação de qualquer reforço e um período de IET 5 s tinha início.

A sessão tinha início com um bloco de 60 tentativas com o estímulo A1 correlacionado com o esquema DRL 1s. Durante a vigência deste esquema, o reforço era apresentado se, no período de 30 s de uma tentativa, o sujeito emitisse duas respostas à barra com, pelo menos, 1 segundo entre a primeira resposta e a segunda (exigência do esquema DRL 1s); caso esta exigência fosse alcançada, o estímulo auditivo era descontinuado, o reforço apresentado e o IET tinha início; caso as respostas ocorressem com menos de um segundo entre elas ou não houvesse a apresentação de uma segunda resposta dentro do intervalo especificado para cada tentativa, o estímulo era descontinuado e seguido por um IET de 5 s.

Quando o critério de obtenção de 90\% dos reforços programados fosse atingido em um dado bloco de tentativas, o bloco subseqüente era programado com DRL 3s, onde o intervalo entre respostas deveria ser de, pelo menos, $3 \mathrm{~s}$ em cada tentativa. Neste esquema, depois que o sujeito emitia sua primeira resposta, qualquer resposta dada depois desta e antes do período de $3 \mathrm{~s}$ postergava o período entre respostas por mais 3 s. Caso suas respostas à barra fossem emitidas com 3 s de intervalo entre elas dentro do período especificado para cada tentativa, o estímulo era descontinuado, o reforço apresentado e o IET tinha início.

$\mathrm{O}$ passo seguinte era a programação de blocos de tentativas programados para exigir um intervalo de $5 \mathrm{~s}$ entre as respostas dentro de cada tentativa (DRL 5s). Mais uma vez, se o sujeito emitisse, em uma tentativa, duas 
respostas com 5 s entre a primeira e a segunda, o estímulo era descontinuado e o reforço apresentado. Caso a segunda resposta fosse emitida em um intervalo menor que $5 \mathrm{~s}$, o período para a emissão de outra resposta era adiado por mais $5 \mathrm{~s}$.

$\mathrm{O}$ treino em DRL 5 s era considerado completo quando o sujeito obtivesse $90 \%$ ou mais dos reforços programados para uma sessão.

Treino do primeiro par. O estímulo A1 (correlacionado com o esquema de reforçamento VR 15) e o estímulo A2 (correlacionado com DRL 5s) eram sucessivamente apresentados em uma mesma sessão experimental. As primeiras sessões eram programadas em blocos de tentativas (60 tentativas de apresentação de A1 correlacionado com VR 15 seguido por 60 tentativas de A2 correlacionado com DRL 5s) até que 360 tentativas fossem completadas. Cada tentativa se iniciava com a apresentação de um dos estímulos auditivos. Quando o estímulo era correlacionado com VR15, as respostas de pressão à barra eram reforçadas de acordo com as contingências descritas acima para o esquema de VR. Quando o estímulo era correlacionado com DRL 5s, as respostas de pressão à barra eram reforçadas de acordo com as contingências descritas acima para o esquema de DRL.

O critério de aprendizagem para esta fase era de $90 \%$ de acertos em uma sessão experimental e, à medida que ele era alcançado, a extensão dos blocos de tentativas diminuía nas sessões seguintes, até que os dois estímulos fossem apresentados em seqüência randômica.

Treino do segundo par. O mesmo procedimento e os mesmos critérios adotados para treinar o primeiro par foram utilizados para treinar o segundo par (B1 correlacionado com VR 15 e B2 correlacionado com DRL 5s).
Treino Conjunto. Após as fases de modelagem e treino das respostas na presença de cada estímulo auditivo, o treino com os quatro estímulos numa mesma sessão foi efetuado. Inicialmente eram apresentados blocos de tentativas (60 tentativas de apresentação de A1 correlacionado com VR 15, 60 de A2 com DRL 5s, 60 de B1 com VR 15 e 60 tentativas de B2 com DRL 5s) até que se completassem 360 tentativas. O número de tentativas por bloco foi sendo gradativamente diminuído $(30,15,10,5)$ até que os quatros estímulos fossem apresentados em uma mesma sessão experimental em ordem randômica. Nas sessôes com seqüência completamente randômica, cada estímulo era apresentado pelo menos uma vez antes que qualquer um deles fosse repetido.

O critério de aprendizagem exigido para considerar que as discriminaçôes foram aprendidas foi de duas sessóes consecutivas com (1) obtenção de $85 \%$ ou mais dos reforços programados para cada estímulo e (2) taxa de resposta, na presença dos estímulos correlacionados com DRL 5 s, igual ou inferior a $50 \%$ da taxa de resposta na presença dos estímulos correlacionados com VR 15.

Após o alcance desse critério, a estabilidade e manutenção da discriminação foram avaliadas em pelo menos 20 sessóes de treino extra. Qualquer queda de desempenho em qualquer uma das sessões de treino para esta fase resultava na programação de sessões de recuperação a mais além das 20 sessões programadas.

\section{Resultados}

Todos os sujeitos concluíram o Treino ao comedouro em uma sessão experimental, bem como a Modelagem de Pressão à Barra. O treino 
gradual do responder adaptado aos dois esquemas de reforçamento foi concluído, em média, em 11,5 sessões para o esquema VR 15 e em 10,2 sessóes para o esquema DRL 5 s.

A Figura 1 mostra a taxa de respostas de todos os sujeitos experimentais, na presença de cada estímulo auditivo nos três treinos: Treino do Primeiro Par, Treino do Segundo Par e Treino Conjunto, da esquerda para a direita respectivamente.

Como pode ser visto nos gráficos à esquerda da Figura 1, todos os sujeitos adquiriram a discriminação A1-VR15 e A2DRL5 durante o treino do primeiro par. As taxas de respostas apresentadas pelos sujeitos eram semelhantes durante o início do treino. A aquisição do responder discriminativo a cada estímulo se deu na medida em que a taxa de respostas na vigência do estímulo correlacionado ao esquema de reforçamento VR aumentava enquanto que a taxa de respostas dos sujeitos experimentais durante a vigência do esquema DRL decrescia ou continuava baixa.
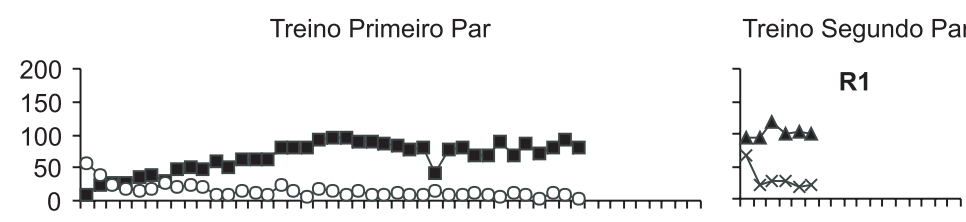

Treino Conjunto
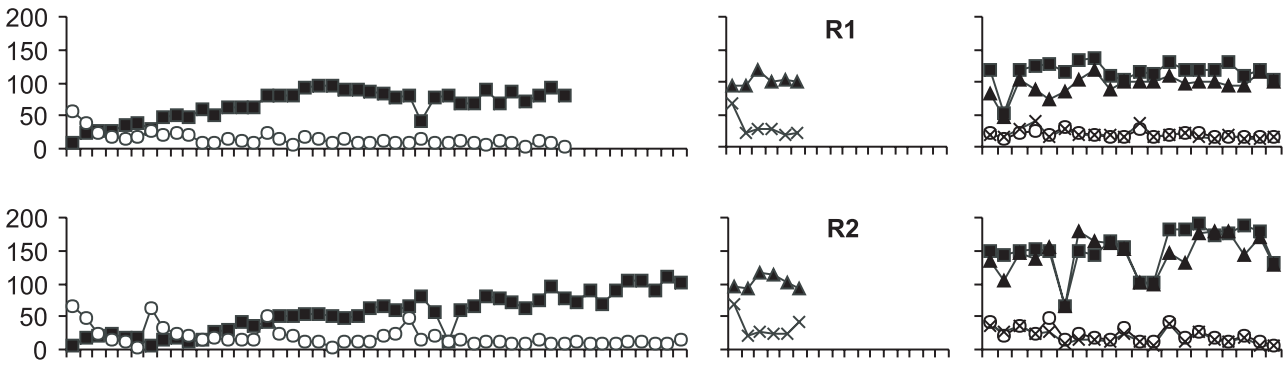

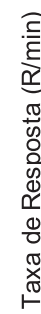

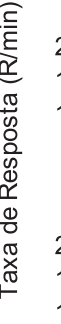
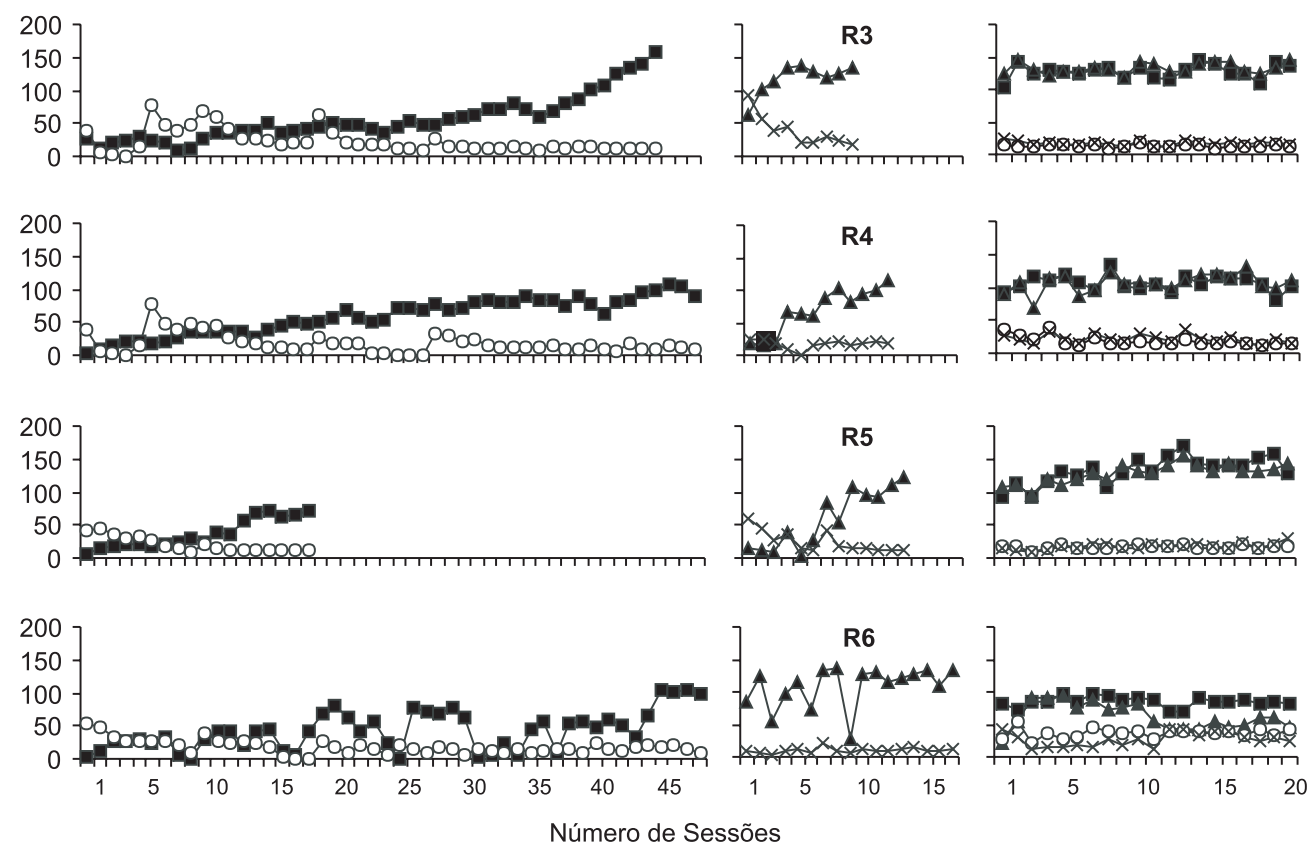

Figura 1. Taxa de respostas $(\mathrm{R} / \mathrm{min})$ de cada sujeito experimental, durante o treino do primeiro par, treino do segundo par e treino conjunto. A seta, na última coluna, indica quando o critério de discriminação foi atingido. 
O número médio de sessões necessárias para que os animais alcançassem o critério para esta fase foi de 41 sessões, pouco menos da metade do que foi necessário para esta etapa no trabalho descrito por Reis (1997). Os sujeitos R2, R4 e R6 passaram por 48 sessões de treino para concluir esta fase, enquanto o Sujeito R5 necessitou de 18 sessões.

A média da taxa de respostas na presença dos estímulos correlacionados ao esquema de reforçamento VR foi de 100,7 respostas por minuto, enquanto que a média da taxa de respostas na presença dos estímulos que sinalizavam DRL 5 s foi de 9,9 respostas por minuto. Mesmo o sujeito com a menor diferença entre as taxas de respostas para os dois esquemas (Sujeito R1) apresentou, ao final do Treino do Primeiro Par, uma taxa de resposta na presença do estímulo que sinalizava o esquema VR 15 de 80,2 respostas por minuto e uma taxa de resposta de 3,1 respostas por minuto na presença do estímulo correlacionado com DRL 5s.

O número de sessões necessário para concluir o treino do segundo par (B1-VR15 e B2-DRL5), coluna central da Figura 1, foi bem menor para todos os sujeitos experimentais quando comparado ao treino do primeiro par (entre 6 e 17 sessões). O Sujeito R5 foi o único exposto a mais que quatro sessões experimentais para apresentar um responder discriminativo aos dois esquemas nesta fase experimental. O Sujeito R6 apresentou, assim como na fase anterior, quedas de desempenho no decorrer das sessôes experimentais e necessitou de maior quantidade de treino até que seu desempenho atingisse o critério. Os demais sujeitos foram submetidos a 8,2 sessões, em média, para alcançar o critério para esta fase experimental. Ainda que esta fase tenha sido concluída mais rapidamente, a taxa de respostas apresentadas pelos sujeitos na presença do estímulo que sinalizava o esquema DRL 5 s foi, em média, o dobro do que a apresentada na fase anterior. A média da taxa de respostas na presença do estímulo correlacionado ao esquema de reforçamento VR foi, para o treino do segundo par, de 99,4 respostas por minuto, enquanto que na presença do estímulo que sinalizava DRL 5s a taxa média foi de 20,7 respostas por minuto.

A manutenção com alta precisão das discriminações A1-VR15, B1-VR15, A2DRL5 e B2-DRL5 foi possível para cinco dos seis sujeitos experimentais durante o treino conjunto. O Sujeito R6 foi o único que não atingiu o critério de índice discriminativo dentro das 20 sessões experimentais programadas para esta fase. O Treino Conjunto para este sujeito foi interrompido após a vigésima sessão por razôes relacionadas à saúde do animal. O desempenho de todos os demais sujeitos experimentais atingiu o critério estabelecido para esta fase já na sétima (indicado por setas em cada gráfico) das 20 sessões de treino conjunto. Para todos os sujeitos, as taxas de respostas na presença dos estímulos sinalizando esquema de reforçamento em DRL 5 s era igual ou menor que $50 \%$ da taxa na presença de cada um dos estímulos designados para sinalizar os componentes em VR 15s. Além disso, todos os sujeitos obtiveram $85 \%$ ou mais dos reforços programados para cada sessão.

Com exceção do Sujeito R6, a taxa de respostas média dos sujeitos para os estímulos correlacionados ao esquema de reforçamento VR foi de 123,6 enquanto que a média da taxa de respostas para os estímulos correlacionados a DRL 5 s foi de 18,6.

Nenhum dos sujeitos necessitou de sessões de recuperação programadas para esta fase, caso 
houvesse queda de desempenho durante o Treino Conjunto. Após o estabelecimento do responder discriminado, a taxa de resposta correlacionado a VR $15 \mathrm{~s}$ foi, no mínimo, três vezes superior a taxa apresentada em DRL $5 \mathrm{~s}$, o que supera em muito os $50 \%$ de diferença entre as taxas exigido no critério.

\section{DISCUSSÃO}

Os resultados mostraram que as mudanças de procedimento introduzidas favoreceram a aquisição das discriminaçôes simples com quatro estímulos auditivos e dois padróes de resposta conjuntos numa mesma sessão. A aquisição das discriminaçôes por cinco dos seis sujeitos parece indicar a efetividade deste treino para o estabelecimento destas tarefas em um período de treino relativamente curto quando comparado ao trabalho de Reis (1997), mesmo com o emprego de sujeitos experimentalmente ingênuos.

Todos os sujeitos experimentais concluíram o experimento em pouco mais de quatro meses. Quando comparado com o trabalho de Reis (1997), isso significa dizer que o emprego de sessōes de longa duração possibilitou a modelagem do mesmo desempenho com um número de sessões de treino três vezes menor. Levando em consideração que ratos são animais de laboratório com vida útil não muito superior a dois anos, este procedimento parece representar um avanço no estabelecimento de uma linha de base rápida para o estudo de classes funcionais em animais deste tipo.

O emprego dos esquemas de reforçamento VR 15 e DRL 5s foi eficiente em gerar taxas de respostas distintas o suficiente para observar padrôes discriminativos particulares a cada conjunto de estímulos sem a necessidade de correlacionar qualquer componente com extinção.

O fato de o número de sessões necessárias para atingir o critério na segunda discriminação (Treino do Segundo Par) ter sido, em média, quatro vezes menor do que o número necessário para concluir a discriminação do primeiro par no Treino do Primeiro Par parece indicar que o treino de novos pares pode ser feito, em estudos futuros, com um número de sessões bem menor do que o relatado na primeira fase do experimento. Desta forma, um próximo estudo poderia planejar o emprego de mais pares de estímulos além dos dois aqui descritos, sem necessitar, entretanto, de um número de sessões de treino muito maior. Este dado parece estar intimamente relacionado ao emprego do treino alternado de responder em VR e DRL ao longo da fase de aquisição dos conjuntos de discriminações, uma vez que ele não é descrito em Reis (1997).

Os resultados foram consistentes com a hipótese de Dube, Callahan et al. (1993) e Harrison (1988), da efetividade do emprego de estímulos sonoros com ratos quando estes são apresentados adjacentes à barra de resposta. Desta forma, o uso de estímulos auditivos passa a representar uma alternativa, juntamente com o emprego de estímulos olfativos (ver Peña, Pitts $\&$ Galizio, 2006), ao uso de estímulos visuais em tarefas de discriminações simples com ratos.

Dos seis sujeitos, apenas o Sujeito R6 não adquiriu as discriminações programadas. Este sujeito veio a falecer um mês depois de seu afastamento do experimento. Os resultados do exame pós-morte indicaram uma infecção generalizada que pode ter contribuído para seu desempenho atípico em todo o experimento.

O fato de a média da taxa de resposta na presença de B2 durante o Treino do Segundo 
Par ter sido muito maior que a taxa apresentada na presença de A2 no Treino do Primeiro Par não pode ser considerado como um evento comum a todos os sujeitos. Esta diferença ocorreu, de fato, somente para o Sujeito R3 que apresentou, durante todas as sessões do Treino do Segundo Par, uma taxa de respostas na presença de B2 muito maior do que a apresentada em A2. Através dos registros visuais das sessões, foi possível verificar que este sujeito apresentava, logo na primeira sessão do Treino do Segundo Par, um responder condizente ao esquema DRL 5s sem respeitar, contudo, o intervalo de 5 segundos entre as respostas no início de cada tentativa. Este padrão fez com que sua taxa de respostas aumentasse para esta fase sem comprometer, todavia, seu desempenho.

A manutenção com alta precisão das discriminações durante todo o Treino Conjunto, sem a necessidade de programação de treinos de recuperação, pode ser considerada como indicação da efetividade deste procedimento em treinar tarefas com elevado índice de manutenção da linha de base das relaçôes ensinadas.

Com o treino discriminativo ocorrendo mais rapidamente, o passo seguinte, em um próximo estudo, seria a adoção do procedimento de reversões repetidas das discriminações em questão. Assim, A1 e B1 poderiam ser correlacionados agora com DRL $5 s$, enquanto que A2 e B2 seriam correlacionados com VR 15 . Uma vez que a nova tarefa fosse aprendida, a contingência poderia ser novamente revertida. As reversões ocorreriam repetidamente até que o contato com as primeiras tentativas possibilitasse ao sujeito inverter a discriminação quando uma reversão fosse feita, como proposto por Vaughan (1988).
Por fim, um teste de formação de classes poderia ser programado onde a função dos estímulos seria revertida somente para um par de estímulos e, uma vez que o critério de aprendizagem fosse alcançado, o par restante seria apresentado. A observação da primeira resposta dos sujeitos direcionada a estes estímulos re-introduzidos no contexto experimental forneceria indícios a respeito da formação ou não das classes funcionais; caso o sujeito respondesse condizentemente a contingência corrente em vigor, isso seria indício de formação de classes funcionais; caso o sujeito respondesse condizentemente com a contingência em vigor antes da última reversão (condição na qual foi feita a última apresentação destes estímulos), isso seria considerado um indício de não formação de classes.

\section{REFERÊNCIAS}

D’Amato, M. R., \& Salmon, D. P. (1984). Tune discrimination in monkeys (Cebus apella) and in rats. Animal Learning and Behavior, 10, 126-134.

de Rose, J. C., McIlvane, W. J., Dube, W. V., Galpin, V. C., \& Stoddard, L. T. (1988). Emergent simple discrimination established by indirect relation to differential consequences. Journal of the Experimental Analysis of Behavior, 50, 1-20.

Dube, W. V., Callahan, T. D., \& McIlvane, W. J. (1993). Serial reversals of concurrent-auditory discriminations in rats. The Psychological Record, 43, 429-440.

Dube, W. V., McIlvane, W. J., Callahan, T. D., \& Stoddard, L. T. (1993). The search for stimulus equivalence in nonverbal organisms. The Psychological Record, 42, 761-778.

Ferrara, M. L, Todorov, J. C., Azzi, R. G., \& OliveiraCastro, J. M. (1983). Desempenhos concorrentes em sessão de longa duração: efeitos de dois valores 
de contingências de mudança. Resumos da XIII Reuniāo Anual de Psicologia da Sociedade de Psicologia de Ribeirão Preto. Ribeirão Preto: SP, Resumo 64.

Ferster, C. B., \& Skinner, B. F. (1957). Schedules of reinforcement. New York: Appleton-Century-Crofts.

Harrison, J. M. (1988). Control of responding by sounds of different quality: An evolutionary analysis. Journal of the Experimental Analysis of Behavior, 50, 521-539.

Iversen, I. H. (1993). Acquisition of matching-to-sample performance in rats using visual stimuli on nose keys. Journal of the Experimental Analysis of Behavior, 59, 471-482.

Iversen, I. H. (1997). Matching-to-sample performance in rats: a case of mistaken identity? Journal of the Experimental Analysis of Behavior, 45, 297-304

Kastak, C. R., Schusterman, R. J., \& Kastak, D. (2001). Equivalence classification by California sea lions using class-specific reinforcers. Journal of the Experimental Analysis of Behavior, 76, 131-158.

Manabe, K., Kawashima, T., \& Staddon, J. E. R. (1995). Differential vocalization in budgerigars: Towards an experimental analysis of naming. Journal of the Experimental Analysis of Behavior, 63, 111-126.

Peña, T., Pitts, R. C., \& Galizio, M. (2006). Identity matching-to-sample with olfactory stimuli in rats. Journal of the Experimental Analysis of Behavior, 85, 203-221.

Reis, M. J. D. (1997). Aprendizagem de reversão parcial ou total em ratos sob discriminação concorrente. Tese de Doutorado não publicada, Universidade de São Paulo, São Paulo.

Reis, M. J. D., \& Costa, T. D. (2002). Discriminação concorrente em sessões de longa duração: uma possível contribuição metodológica. Arquivos Brasileiros de Psicologia, 54, 304-317.

Schusterman, R. J., \& Kastak, D. (1993). A California sea lion (Zalophus californianus) is capable of forming equivalence relations. The Psychological Record, 43, 823-839.

Sidman, M. (1971). Reading and auditory-visual equivalence. Journal of Speech and Hearing Research, 14, 5-13.

Sidman, M. (1994). Equivalence relations and behavior: A research story. Boston, MA: Authors Cooperative, Inc., Publishers.

Sidman, M. (2000). Equivalence relations and the reinforcement contingency. Journal of the Experimental Analysis of Behavior, 74, 127-146.

Sidman, M., \& Tailby, W. (1982). Conditional discrimination vs. matching-to-sample: An expansion of the testing paradigm. Journal of the Experimental Analysis of Behavior, 37, 5-22.

Vaughan Jr., W. (1988). Formation of equivalence sets in pigeons. Journal of Experimental Psychology: Animal Behavior Processes, 14, 36-42.

Submetido em 13 de novembro de 2006 Aceito em 15 de novembro de 2007 


\section{INSTITUTO BRASILIENSE DE ANÁLISE DO COMPORTAMENTO}

\section{WWW.IBAC.COM.BR}

Curso de Especialização em Análise Comportamental Clínica Carga Horária: 540h - Duração: 2* a 3 anos - conforme opção do aluno

Turmas semestrais - Aulas sábado pela manhã

Clientela: graduados em psicologia

Regulamentado pelo CFP - ABEP

Curso de Especialização em Terapia Analítico Comportamental Infantil Carga Horária: 500h - Duração: 2 anos

Turmas a cada 2 anos - Aulas um final de semana por mês

Clientela: graduados em psicologia

Em processo de regulamentação pelo CFP-ABEP

Curso de Formação em Análise Comportamental Clínica

Carga Horária: 180h - Duração: 1 ano e 1/2 ou 2 anos - conforme opção do aluno

Turmas semestrais - Aulas sábado à tarde

Clientela: graduandos em psicologia a partir do $6^{\circ}$ semestre, psicólogos

Curso de Formação em Terapia Analítico Comportamental Infantil

Carga Horária: 180h - Duração: 1 ano e 1/2 -

Turmas semestrais - Aulas sábado à tarde

Clientela: graduandos em psicologia a partir do $7^{\circ}$ semestre, psicólogos

Inglês instrumental para psicólogos

Grupos de Estudos em Análise do Comportamento

Terça-Clínica: Discussão de casos clínicos em AC

Cinemateca IBAC

Simpósio Brasiliense de Análise Comportamental Clínica 